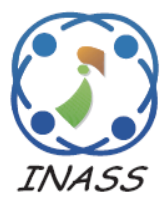

\author{
International Journal of \\ Intelligent Engineering \& Systems
}

http://www.inass.org/

\title{
A Streamlined OCR System for Handwritten Marathi Text Document Classification and Recognition Using SVM-ACS Algorithm
}

\author{
Surendra Pandurang Ramteke ${ }^{1 *}$ \\ Ajay Anil Gurjar ${ }^{2}$ \\ Dhiraj Sheshrao Deshmukh ${ }^{3}$ \\ ${ }^{I}$ Department of Electronics \& Telecommunication Engineering, \\ Shram Sadhana Bombay Trust College of Engineering and Technology, Bambhori, Maharashtra, India \\ ${ }^{2}$ Sipna College of Engineering \& Technology, Maharashtra, India \\ ${ }^{3}$ Department of Mechanical Engineering, \\ Dr. Babasaheb Ambedkar College of Engineering \& Research, Maharashtra, India \\ * Corresponding author's Email: rsurendra0711@gmail.com
}

\begin{abstract}
Handwritten optical character recognition (OCR) is a noteworthy research region because of its sensitivity in segmenting the character which increments on account of MARATHI script because of modifiers and compound characters. This paper gives a streamlined OCR framework for handwritten MARATHI text document classification and recognition system. To deal with a vast measure of features, the support vector machine (SVM) assumes a noteworthy part which was likewise used for the classification reason. In this paper, we display a projection profile segmentation technique which generates less error. The Curvelet Transform (CT) to be exceptionally efficient and hearty to get the feature characters from the pre-processed image. The extracted feature sets are decreased by Principle Component Analysis (PCA) algorithm. After the feature extraction process, the Adaptive Cuckoo Search (ACS) algorithm is used for the optimization procedure. Here, the written by hand MARATHI script was segmented flexibly in three levels; (1) line segmentation, (2) word segmentation and (3) character segmentation. The preprocessing was finished utilizing different morphological operations. The experimental results show that, the performance of the proposed technique is assessed in view of the accuracy, sensitivity, precision, recall and F-score. Compared with the existing Fire Fly Selection (FFS) and Bat Selection (BS) approach, the proposed method has 99.36\% accuracy, 90\% sensitivity, 91\% precision, $89.51 \%$ recall, $99.67 \%$ specificity and $89.93 \%$ F-score. The proposed approach is actualized using MATLAB and the realtime Marathi character datasets are used for our examination.
\end{abstract}

Keywords: Optical character recognition (OCR), Curvelet transform (CT), Principle component analysis (PCA) algorithm, Adaptive cuckoo search (ACS) algorithm, SVM for classification and recognition.

\section{Introduction}

All Optical Character Recognition (OCR) especially of English language documents has been extensively studied and implemented successfully over a number of years [1]. Devanagari script is used for the majority of Indian languages, like Hindi, Marathi, Sindhi, and Sanskrit etc. Some of the Indian scripts like Guajarati, Punjabi, and Kannada etc. to have been derived from the Devanagari script $[2,3]$. Handwritten characters have an infinite variety of styles from one person to another person.
Due to this wide range of variability, it is difficult to recognize by a machine $[4,5]$. Most of the researchers have tried to solve the problems based on the image processing and pattern recognition techniques [6-8].

OCR methodologies can be classified based on two criteria; data acquisition process which can be on-line or off-line and type of the text which is printed text or hand-written text $[9,10]$. Both the tasks are challenging for automatic character recognition, specifically in off-line character recognition requires more efforts due to various reasons viz [11-13]. 
Many of the systems have elaborated about a variety of different techniques for text character segmentation; they are; A Support Vector Machine (SVM), k-NN based classifier [14, 15]. Wavelet transforms [16-18] and neural network classifier [19] to recognize handwritten Tamil pattern recognition. In the Devanagari script, to identify the Marathi numerals, a discrete cosine transform and neural networks are utilized in [20]. This approach gives better results, however, the neural network includes greater computational burden in the character classification. Compared with other classification methods, it generates only $90.30 \%$ accuracy. The above mentioned techniques are not proven reliable for the optical character recognition.

The fundamental goal of our present work is to plan a streamlined OCR system for handwritten Marathi text document classification and recognition using Support Vector Machine (SVM) and Cuckoo Search (CS) algorithm. SVM is a binary classification algorithm; however, it is used to classify the feature database of the document. CS is based on the Levy flight behavior of some birds and fruit flies combined with the obligate brood-parasitic behavior of some cuckoo species. To optimize the SVM, the CS algorithm is used to pick out the Marathi text from the handwritten document. For this classification and recognition the preprocessing, segmentation, feature extraction and dimensionality reduction techniques are proposed in our method.

Compared with the existing method, the proposed approach would add to decrease the computational costs, so our proposed method accomplishes high accuracy and furthermore, this approach accurately recognizing the substantial volume of characters.

Whatever is left of the portion of the paper is delineated in the section underneath. In segment 3, the preprocessing techniques and the feature extractions are portrayed. The proposed SVM classification technique and the ACS algorithm are portrayed in section 3.6 and 3.7. The results and the conclusions are portrayed in segment 4 and 5 .

\section{Literature survey}

Karbhari et al. [13] have presented a Zernike moment feature descriptor and implemented SVM and k-NN based classification system for character recognition. This introduces some errors since it involves the re-sampling and re-quantifying of digital images. The proper recognition of Devanagari characters using a variation of Gradient, Structural features and artificial neural network

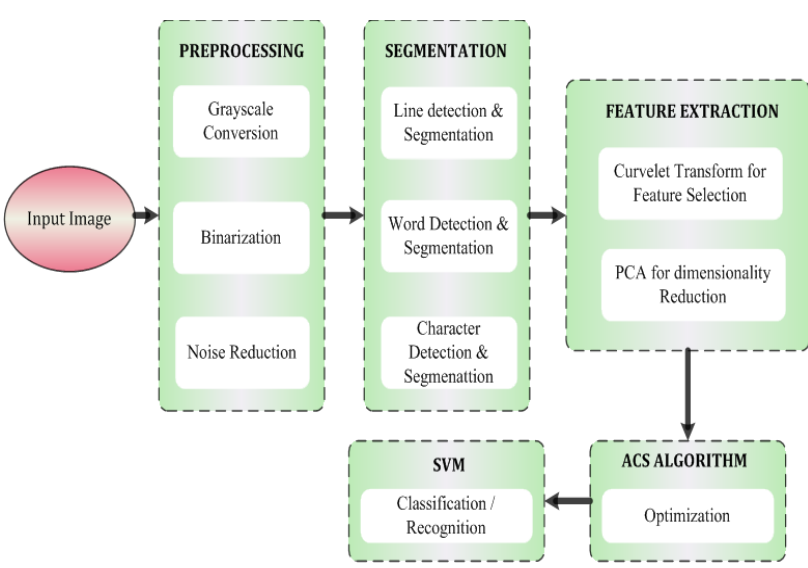

Figure.1 Optical character recognition system

(ANN) was proposed by Abdalkafor [14]. It may suffer from some overfitting problems.

P. Kamble and R. Hegadi [15] have proposed the Rectangle Histogram Oriented Gradient representation as the basis for extraction of features. It can be quite sensitive to over-fitting the model selection criterion. P. Singh et al. [16] have presented a Multi-Layer Perceptron (MLP) classifier for Optical Character Recognition (OCR). Their method sensitive to feature scaling. S. Naz et al. [17] have employed the optical character recognition (OCR) literature with reference to the Urdu-like cursive scripts. However, the computational cost is high.

J. Olszewska [18] presented a new optical character recognition (OCR) approach. The main drawback is sensitive to local minima states. O. Matei et al. [19] have proposed a novel process to optical character recognition (OCR). In their method, the main drawback of $\mathrm{KNN}$ is memory limitation, it is sensitive to the local structure of the data, supervised lazy learner and it runs slowly.

\section{Proposed method for optical character recognition (OCR) and classification}

Fig. 1 shows the architecture of the proposed OCR. Initially, the text documents are converted into the image samples in the preprocessing stage. In the wake of pre-processing, the curvelet transform is connected to extricate the statistical distance profile feature and the gradient features of the preprocessed image. A typical feature of handwritten text is the introduction of text composed by the author. The curvelet transform at a single scale is connected to each of the samples to get curvelet coefficients as features. Feature extraction stage is to expel redundancy of data. The extracted feature sets are reduced by the PCA algorithm. At that point the segmentation stage is done to build the proficiency for the next stage. The segmented feature sets are 
given as the contribution to the classification process. The SVM classifier and the CS optimization algorithm should use for the classification and optimization reason to recognize the Marathi word image.

\subsection{Data acquisition}

In the image acquisition process, the handwritten or the paper documents are converted into electronic form or the picture form. In our method, the image is processed from the handwritten document by using the scanning process.

\subsection{Pre-processing}

The image to be pre-processed after the image acquisition process, here, to get the best quality of image is occurring through the noise reduction and image enhancement step. The input image is initially converted to the readable format, if the image is not in the proper format. The pre-processing steps include the following steps to pre-processing the image of Fig. 2.

(a) Grayscale Conversion: Initially the scanned images is contained color character images such as RGB (Red, Green, and Blue) format and it is stored in the BMG, JPEG and TIFF etc. So these images are converted to gray scale image format. Here, the images are present in the matrix form where all the value of the every element is equivalent to how bright or dark the pixel at the appropriate position should be colored.

(b) Thresholding: In this process, the grayscale image is converted into a binary image by using the thresholding procedure. For reducing the required storage space and increase the rate of processing. These are done based on the threshold value.

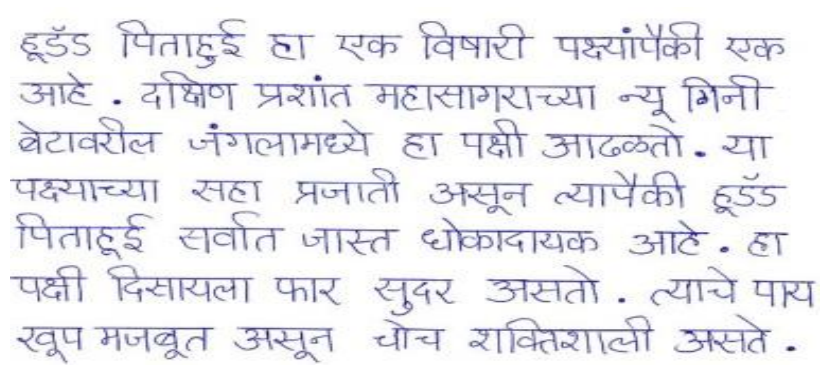

Figure.2 Pre-processed image (c) Noise Reduction: The disconnected line segment, gaps and bumps in lines are caused by the scanning devices these devices generate the noises in the image. The local variations, dilation and erosion, etc., are the main distortions and also it is very pivotal to supplant the restrictions. The filtering, morphological operation and noise modelling is the important techniques in the noise reduction process.

\subsection{Segmentation}

In the segmentation process, the pre-processing image is partitioned into a number of regions. The Local segmentation and global segmentation are the main two types of the segmentation process. The sub images are dealt with the local segmentation and the whole image is dealt with using the global segmentation process.

In the global threshold process, the threshold level is considered based on the intensity values of the image. If the intensity value is less than the threshold level is set 0 (black); the rest are set 1 (white). Here, the global threshold of the image $f(a, b)$ is represented as $T$ and the threshold image $g(a, b)$ is given as follows,

$$
g(a, b)= \begin{cases}1, & \text { if } f(a, b) \geq T \\ 0, & \text { otherwise }\end{cases}
$$

In our method, the projection profile method is utilized for the segmentation technique. The segmentation technique incorporates the three important techniques, they are;

- Line detection and Segmentation

- Word detection and Segmentation

- Character detection and Segmentation

(a) Line Detection and Segmentation: In this detection and segmentation method, initially the sum of all white pixels on every line or row is estimated by utilizing the horizontal projection profile method and also it generates the appropriate histogram of the image.

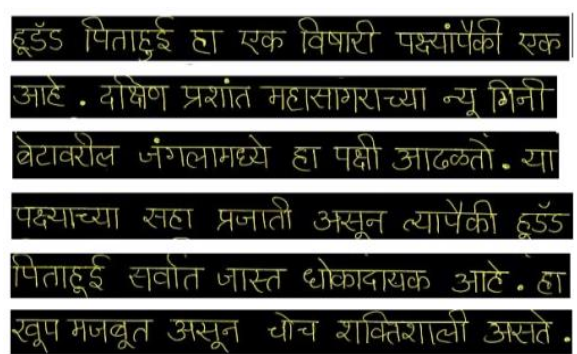

Figure.3. Segmented lines 


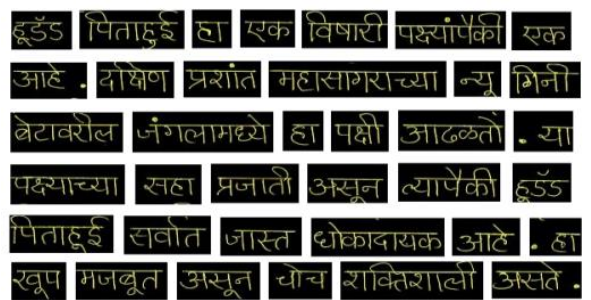

Figure.4. Segmented words

- Constructs the horizontal histogram of the image.

- Find out the distance between the appropriate two histograms which is the threshold value.

- Based on the threshold value separate every histogram and save it.

- Finally, the segmented line is generated from the image.

(b) Word Detection and Segmentation: The vertical projection profile method is utilized to estimate the sum of all white pixels and the estimated white pixel's histograms are plotted. The segmentation of the word is depicted as Fig. 4 ,

- Constructs the vertical histogram for the image.

- In each column, find out the number of white pixels (threshold) and then find out the columns containing no white pixel by utilizing the histogram.

- Replace all such columns by 1 and convert the empty rows as 0 and text words will have original pixels and save it.

- Based on the threshold value, the words are segmented from the line.

- Repeat the process for each line.

(c) Character Detection and Segmentation: Both the horizontal and the vertical projection file methods are utilized in the character segmentation process and it separates the characters from the words. The character segmentation steps are depicted as follows,

- Construct both the horizontal and the vertical histogram for the image.

- Find out the number of white pixels and plot the histogram for the estimated pixel.

- Find out the upper horizontal line or shirorekha and supplant it.

- Find out the threshold value and separate the histogram by utilizing the vertical histogram

- Based on the threshold values the characters are segmented from the words and save it.

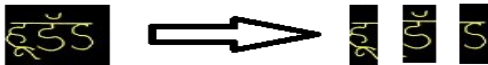

Figure.5. Segmented characters

\subsection{Curvelet transform for feature extraction}

The Marathi language incorporates a large set of characters, so recognize these characters accurately an automatic curve matching is highly efficient. So we utilize a curvelet transform for the extraction of variety of image characteristics, which can accurately represent the edge of the image to a various scales and various frequency sub-bands. In image feature extraction, the curvelet transform has high potential. In the Marathi document classification and recognition, many of the characters in a word incorporate both the edge discontinuities and curve discontinuities. For the edge discontinuity, the Wavelet transform is a well known method, but, it does not utilize for the curve discontinuity. But the curvelet transform can handle the curve discontinuities in any character or word. For the Marathi character and word recognition, the curvelet transform is working very efficiently. The main steps of the curvelet transform are, (1) Subband decomposition, (2) Smooth partitioning, (3) Renormalization and (4) Ridgelet analysis.

(a) Sub-band Decomposition: Let us consider $f(a, b)$ as the Marathi text image array and these images are converted into a number of layers or sub-bands and every converted layers or sub-bands contains the different frequencies and it is given as follows,

$$
f \rightarrow\left(L_{p_{0}} f, \Omega_{1} f, \Omega_{2} f \mathrm{~K}\right)
$$

In the above equation, the low pass filter is represented as $L_{p 0}$ and the high pass or the band pass filter is represented as $\Omega_{1}$ and $\Omega_{2}$.

(b) Smooth partitioning: In the smooth partitioning process, the initially converted sub-bands are windowed into squares of an appropriate scale and it is given as follows,

$$
\Omega_{s} f \rightarrow\left(u_{D} \Omega_{s} f\right)_{D=D_{s}}
$$

In the above equation, the dynamic square of the side $2^{-s}$ is represented as $D_{s}$ and the smooth windowing function represented as $u$ and the size of the dynamic square is $2^{-s} \times 2^{-s}$. 
(c) Renormalization: In this step, the previous resulting square is renormalized to unit square and it is given as follows,

$$
R_{D}=2^{-s}\left(T_{D}\right)^{-1}\left(u_{D} \Omega_{s} f\right), D \in D_{s}
$$

(d) Ridgelet analysis: Each square is analyzed in the ortho-ridgelet system and it is given as follows,

$$
x_{\mu}=\left\langle R_{D}, \delta_{\eta}\right\rangle, \mu=(D, \eta)
$$

In the above equation, the ortho normal basis is represented as $\delta_{\eta}$. The reverse process of the decomposition process is the synthesis step of the Curvelet transforms. Initially, the Ridgelet synthesis performed,

$$
R_{D}=\sum_{\eta} x(\eta, D) \delta_{\eta}
$$

The renormalization is given as follows,

$$
h_{D}=\left(T_{D}\right)_{R_{D}}, D \in D_{s}
$$

The smooth integration for blocks of each sub-bands are given as follows,

$$
\Omega_{s} f=\sum_{D \in D_{s}} u_{D} \cdot h_{D}
$$

At last the reconstruct sub-bands are given as follows,

$$
f=L_{P_{0}}\left(L_{P_{0}} f\right)+\sum_{s>0} \Omega_{s}\left(\Omega_{s} f\right)
$$

In the wavelet transform, the information about the edges are lacking in some expression. These shortcomings of the wavelet transform are solved by the above depicted descriptions.

\subsection{PCA system for dimensionality reduction}

After the feature extraction process, the Curvelet transform generates the large dimensional feature space; this is the significant problem by utilizing the Curvelet transform. If the feature space is large, lots of training data are required by the classifier, so it consumes more time to classify the data. So, the dimensionality reduction is the obvious choice, so we will utilize the PCA method for the feature space reduction process. In the PCA method, the eigenvectors of the covariance matrix are estimated for all the original input. Here, a set of possible correlated variable is converted into linear uncorrelated variables, for this process the orthogonal transformation function is utilized by the PCA. Initially, the eigenvalues are generated from the previously generated feature matrix, and then the very little eigenvalues are supplanted in the sorting method. Then the data is transformed into the eigenspace which is formed by the selected eigenvectors.

\subsection{Adaptive cuckoo search (ACS) algorithm for optimization}

The Cuckoo Search (CS) algorithm is the very best means of optimization in such problems. For developing new optimization algorithm, this cuckoo breeding anatology is utilized. CS algorithm is based on the Lévy flight behavior of some birds and fruit flies combined with the obligate brood-parasitic behavior of some cuckoo species. In the CS algorithm, as per the law of Levy flight, the new nest is generated in the environment. In the environment, the birds searching for food are one of the ways of motion used by them, which is represented by the Lévy flight to tune the parameter of the input in OCR system; a modified CS algorithm is suggested in this document. Based on the Levy flight bahavior, the better points replaced in the current population and a new population at each generation are created. In the case of error character minimization problems, the quality or fitness of a solution can simply be proportional to the minus value of the objective function.

Initialization of the feature extracted solutions of the OCR system is done here. The function which is denoted as $A_{i}(t)$ is considered for the initialized parameters. The randomized solution is generated from the initialized values and the following describes the initialized value.

$$
A_{i}=\left[a_{0}^{i}, a_{1}^{i}, \Lambda, a_{N_{L-1}}^{i}\right]
$$

Where, the random value which incorporates the input is $A_{i}$ and the length of the value is $N_{L}$.

From the initialized values the current best nest is calculated during evaluation. In the next generation of time $(t+1)$, the new solution is represented as,

$$
A_{i}(t+1)=A_{i}(t)+\alpha \oplus \operatorname{Levy}(\lambda)
$$


Where, the entry wise multiplication is denoted by $\oplus$, the step size is $\alpha$ and the levy distribution is Lev' $y(\lambda)$. In most of the cases, the value of $\alpha$ is being equal to 1 .

\section{Step 1: Initialization \\ The $N$ number of host nests $A_{i}$ are randomly generate, for $i=1,2, \ldots, N$, which is represented in eqn (10). Initially assign $t=1$ estimate the fitness function $f\left(A_{i}\right)$ for the host nests.}

Step 2: Iterative algorithm

Step 2.1: Estimate the best $(f)$ and wost ( $f$ ) for the current generation among the hosts nests by performing Levy flights.

Step 2.2: Estimate the step size by using the following equation,

$\operatorname{Step}_{i}(t+1)=(1 / t)^{\left.\mid\left(\text {best } f(t)-f_{i}(t)\right) /(\text { best } f(t)-\text { worst } f(t))\right) \mid}$

Step 2.3: Estimate the new position of Cuckoo nests using the following equation

$A_{i}=(t+1)=A_{i}(t)+\operatorname{randn} \times \operatorname{step}_{i}(t+1)$

Step 2.4: Estimate the fitness function of the hosts nests $f\left(A_{i}\right)$ for $i=1,2, \ldots, N$.

Step 2.5: After that randomly select nest $j$ for all $N$

Step 2.6: If the fitness function $f_{i}$ is greater than the newly generated fitness function $f_{j}\left(f_{i}>f_{j}\right)$, update the $j-t h$ nest by the new solution

\section{End}

Step 2.7: The best solutions are built and the worst nests are forsaken and set $t=t+1$

Step 2.8: If $t_{\max } \geq t$, repeat the process from

step 2.1: otherwise end.

After that the best solutions are reported by ranking them.

Figure.6 Algorithm steps for adaptive cuckoo search (ACS) algorithm

Based on the inputs, the output of the function is evaluated in the step. Determination of the minimized error is done. Randomly the location is turned by the cuckoo and the fitness function is evaluated. Otherwise, a randomly selected coordinates of an egg are used to perform the Levy flight to generate a new egg in CS. Most commonly, the Mantegna algorithm is utilized to generate the Levy distribution. This can be represented as follows,

$$
\operatorname{Levy}(\lambda)=\frac{v}{|y| \frac{1}{(u-1)}}
$$

Where, $v$ and $y$ are generated from a normal distribution, the range of $\lambda$ is considered as [1,3] and the standard deviation related to the normal distribution is represented as,

$$
\omega_{n}(\lambda)=\left[\frac{\Lambda(1+\lambda) \sin \left(\frac{\pi \lambda}{2}\right)}{\Lambda\left(\frac{1+\lambda}{2}\right) \lambda^{2}}\right] \text {, and } \omega_{n}(\lambda)=1
$$

For updating the Levy flight function of CS algorithm, the adaptive CS (ACS) algorithm is utilized in this part. For improving the performance of the CS algorithm ACS is updated.

To control the step size in the iteration process, the Cuckoo search algorithm does not have any mechanism, which can guide the algorithm to reach global minima or maxima. In this step, a step size is implemented which is proportional to the fitness of the individual nest in the search space in the current generation. In the adaptive CS algorithm, the step size is modeled as,

$$
\operatorname{step}_{i}(t+1)=\left(\frac{1}{t}\right)^{\left|\frac{\left(\left(\text { best } f(t)-f_{i}(t)\right)\right)}{(\text { best } f(t)-\text { worst } f(t))}\right|}
$$

In the above equation,

$t \rightarrow$ Generation of Cuckoo search

$f_{i}(t) \rightarrow$ Fitness vale of the I-the nest in the $\mathrm{t}$-the generation

best $f(t) \rightarrow$ Best fitness value in $\mathrm{t}$-th generation

worst $f(t) \rightarrow$ Worst fitness value in $\mathrm{t}$-th generation

At first the step size is high; if the number of generations increases the step size will be decreased. This means that the step size is very small, when it attains the global optimal solution. New position of cuckoo nests is estimated in this step. From Eq. (14) we found that the step size resolves its value from the fitness value, so step size is flexible in nature. Thus, displayed the adaptive CS algorithm as:

$$
A_{i}(t+1)=A_{i}(t)+\operatorname{randn} \times \operatorname{step}_{i}(t+1)
$$

From the current solution the Eq. (15) gives the new search space for the ACS. It does not require any initial parameters to be defined; it is another advantage of the ACS. It is faster when compared to the cuckoo search algorithm. Further, when the step 
size is considered to be proportional to the global best solution, at that point Eq. (14) is displayed as:

$$
\begin{gathered}
A_{i}(t+1)=A_{i}(t)+{\text { randn } \times \operatorname{step}_{i}(t+1) \times} \\
\left(A_{i}(t)-A_{\text {gbest }}\right)
\end{gathered}
$$

In the above equation, the global best solution is represented as $A_{\text {gbest }}$ at time $t$. The process is repeated until it satisfies the condition by checking the final solution at the end of maximum iteration.

\subsection{Classification and recognition based on SVM classifier}

In this work, the SVM classifier is implemented for the classification which takes the output of the optimized output of the feature extraction techniques to their input and then determine which class is actually belong to. The SVM is a powerful supervised classifier and an accurate learning technique. The main goal of the SVM classifier is classification error minimization and discrimination margin maximization.

The optimized feature vectors of the training set $A$ are considered as $A_{i}, i=1,2,3, \ldots, N$. The training set belongs to any one class of the classes $\delta_{l}$ and $\delta_{l}$. To find an optimal hyper plane, these two classes are separated by the SVM utilizes the training data. The hyper plane can be computed as follows,

$$
h(A)=Z^{T} A+Z_{0}=0
$$

In this work, four different kernel functions are utilized, they are; linear, polynomial, Radial Basis Function (RBF) and sigmoid and it is given as follows,

The linear kernel equation is given by,

$$
K\left(A, A_{i}\right)=A_{i}^{T} A
$$

The polynomial kernel is given as follows,

$$
K\left(A, A_{i}\right)=\left(1+\frac{a_{1}^{T} a}{c}\right)^{d}
$$

The RBF is computed as follows,

$$
K\left(A, A_{i k}\right)=\left(\frac{-\|a-1\|}{\sigma^{2}}\right)
$$

The sigmoid kernel function is computed as follows,

$$
K\left(A, A_{i}\right)=\tanh \left(K a_{i}{ }^{T} a+\varphi\right)
$$

The scaling of the inputs in the kernel functions is represented as $c, \sigma$, and $i$. The SVM classifier takes the contribution from the as of now created feature vectors and a learning model is acquired as yield toward the finish of this stage. The Linear kernel is a very simple method and estimated very quickly, so, our SVM classifier utilized the Linear Kernel, it is very pivotal to select a value for the soft margin in advance. After that, the optimized feature vector $a_{i}$ of the training data considered in the class $c_{i} \in[-1,1]$, the SVM solves,

$$
\min _{\alpha, \beta, \chi} \frac{1}{2} K(\varpi, \varpi)+\sigma \sum_{\alpha=1}^{1} \alpha_{i}
$$

Subject to $c_{i}\left(K\left(\varpi, \alpha_{i}\right)+b\right) \geq 1-\alpha_{i} \quad \alpha_{i} \geq 0$

In the above equation, a 1-dimensional vector is represented as $\alpha$, in feature space $\alpha_{i}$, the vector is represented as $\varpi$. In the original feature space, the hyper plane is determined by using the values $\varpi$ and $b$. To obtain the best performance on our selected portion of the data, we will optimize this choice.

\section{Results and discussions}

The performance evaluation utilizing SVM based classifier has been performed in view of the real time dataset of handwritten Marathi characters. Materials of the handwritten documentation pages for the proposed databases have been composed by different people. These handwritten documents are gathered from various people who were expected to compose printed substance browsed the daily paper articles or books containing Marathi vocabularies.

\subsection{Dataset descriptions}

A self created database was utilized in this research. Here there are 49 Marathi characters are utilized for the testing and the training process. The whole system was implemented in MATLAB. After the preprocessing stage, all the data samples are divided into two parts: 56100 data samples are reserved for training purpose while 9900 data samples are reserved for testing purpose. 


\subsection{Evaluation metrics}

The segmentation and the classification results are compared to the existing segmentation techniques. Each label of the data samples consists of true positive (TP), true negative (TN), false positive (FP) and false negative (FN) classes. The the handwritten Marathi characters are correctly classified by these four classes. Here, TP is the positive label that was correctly labeled by the classifier. $\mathrm{TN}$ is the negative labels that were correctly labeled by the classifier. FP is the negative labels that were incorrectly classified by the classifier and FN is the positive labels that were incorrectly labeled as negative. To evaluate the classification results there are three quantitative metrics are utilized. They are delineated in the following equations,

$$
\begin{gathered}
\text { Precision }=\frac{T P}{T P+F P} \\
\text { recall (or) specificity }=\frac{T P}{T P+F N}
\end{gathered}
$$

Fig. 7 demonstrates the precision of the proposed strategy. Here, the number of test characters is 49 and the proposed method has better precision when compared with the existing character recognition approach. The specificity examination of the proposed strategy appearing in Fig.8. Here, the graph is plotted for the precision of the proposed method with respect to the total number of test characters. To evaluate the performance of the classifier, (i.e.), if the Marathi character is correctly classified or not is evaluated by the following metrics,

$$
\begin{aligned}
& \text { Sensitivit } y=\frac{T P}{T P+F N} \\
& \text { Accuracy }=\frac{T P+T N}{T P+T N+F P+F N} \\
& F-\text { Mscore }=\frac{2 \times \text { Precision } \times \text { Recall }}{\text { Precision }+ \text { Recall }}
\end{aligned}
$$

Fig. 9 demonstrates the sensitivity of the proposed strategy. Here, the graph is plotted for the sensitivity of the proposed method with respect to the total number of test characters.

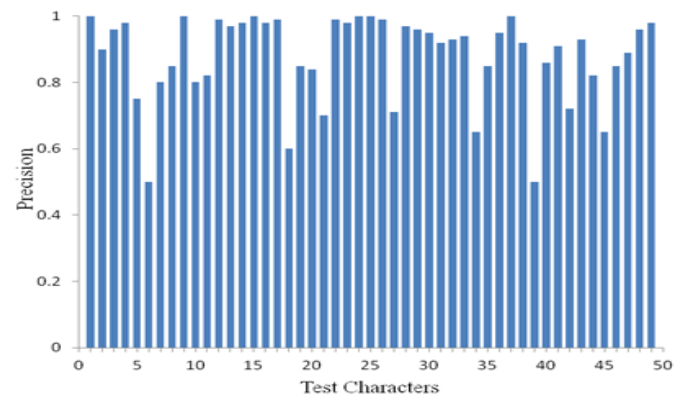

Figure.7 Precision of the proposed method

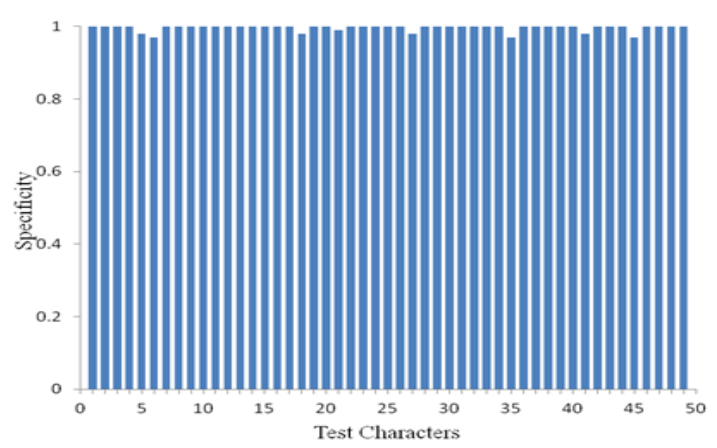

Figure.8 Specificity of the proposed method

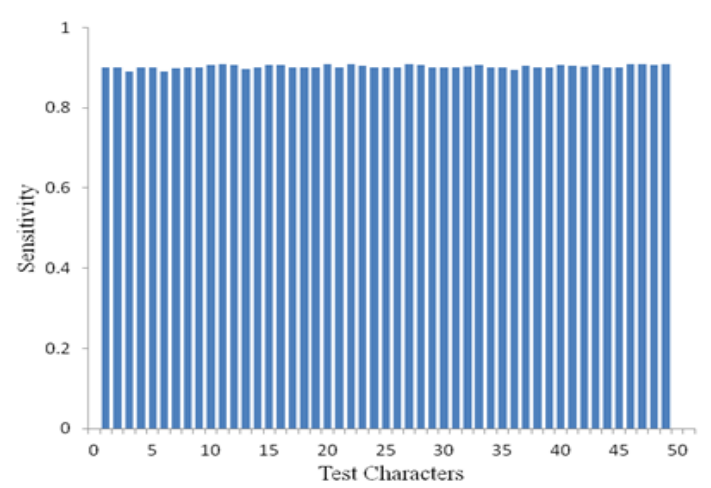

Figure. 9 Sensitivity of the proposed method

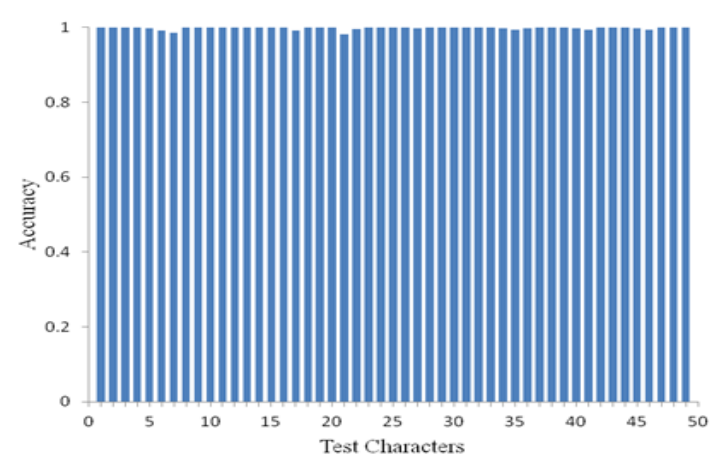

Figure.10 Accuracy of the proposed method

Fig.10 demonstrates the accuracy of the proposed strategy. The proposed method has better accuracy and sensitivity when compared with the existing character recognition method.The execution of the framework depends on the F-measure esteems, which can be appeared in Fig.11. The F-score is the combination of the precision and recall. 


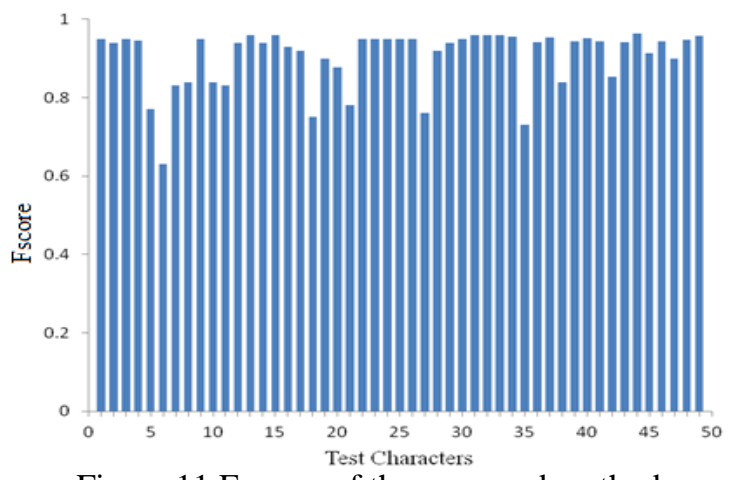

Figure.11 F-score of the proposed method

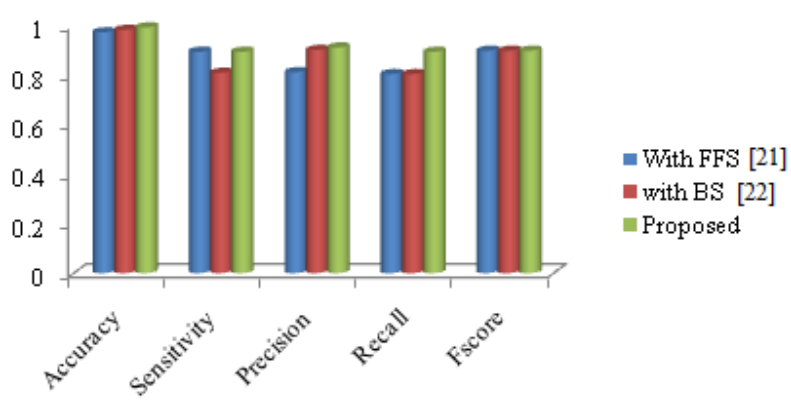

Figure.12 Comparative analysis of the proposed method

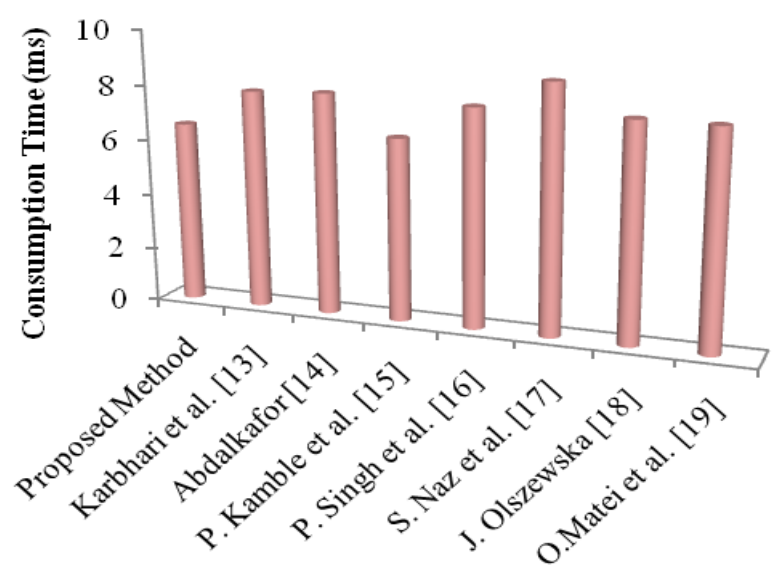

Figure.13 Comparison Results for with other methods

\subsection{Comparison analysis}

Fig.12 demonstrates the comparison result of the proposed technique. The accuracy of the proposed method and the existing methods are almost same. Compared with the FFS (Fire Fly Selection) [21] and the BS (Bat Selection) algorithm [22], the proposed method has the better sensitivity and recall. Because, FFS algorithm is a bio-inspired optimization algorithm and the optimization problems are accurately solved by this algorithm. However, the FFS can easily get trapped in the local optima and cause low precision. In the Bat selection approach, the performance of BS is largely dependent on the parameters of the algorithm, sometime inefficiently parameter controls and low convergence speed. Theses drawbacks are overcome by the proposed ACS algorithm. Because the ACS algorithm is a parameter free algorithm, so the convergence speed is high.

Fig.13 demonstrates the comparison results of other methods like Zernike moment method [13], Artificial Neural Network [14], Support Vector Machine and Feed Forward Artificial Neural Network (SVM-FFANN) [15], MLP (Multi Layered Percerptron) [16], Fuzzy logic [17], Active contour based method [18], Neural Network [20]. These existing methods are compared with the proposed Marathi character recognition approach. Here, the consumption time of the proposed method is better (6.55 (ms)) than the existing method. The consumption time of the existing method is 7.89 (ms), 7.99 (ms), 6.59 (ms), 7.85 (ms), 8.87 (ms), $7.78(\mathrm{~ms})$ and $7.75(\mathrm{~ms})$.

\section{Conclusion}

The experimental result represents that the Support Vector Machine (SVM) technique can be connected effectively to fathom the Marathi optical character recognition issue. The reason for our work is to think about the performance of different classification techniques with different feature extraction and segmentation methods. Our approach would add to lessen the computational expenses, so our proposed method accomplishes high accuracy and furthermore, this approach accurately recognizing the substantial volume of characters. The experimental results show that the proposed method has the accuracy is $99.36 \%$ and the consumption time is $6.55(\mathrm{~ms})$

In future work the classification rate additionally can be enhanced by applying other clustering or classification systems like fuzzy neural network and genetic algorithm. Additionally, another feature extraction procedure can be connected for better accuracy.

\section{References}

[1] P. Roy, A. Bhunia, A. Das, P. Dey, and U. Pal, "HMM-based Indic handwritten word recognition using zone segmentation", Pattern Recognition, Vol. 60, No. 12, pp. 1057-1075, 2016.

[2] P. Yadav and S. Sharma, "Enhancing Performance of Devanagari Script Recognition using Hopfield ANN", International Journal of Engineering Trends and Technology, Vol. 36, No. 2, pp. 66-75, 2016.

[3] A. Choudhary, R. Rishi, and S. Ahlawat, "Offline Handwritten Character Recognition Using 
Features Extracted from Binarization Technique", AASRI Procedia, Vol. 4, No. 1, pp. 306-312, 2013.

[4] N. Garg, L. Kaur, and M. Jindal, "Segmentation of touching modifiers and consonants in middle region of handwritten Hindi text", Pattern Recognition and Image Analysis, Vol. 25, No. 3, pp. 413-417, 2015.

[5] R. Sarkhel, N. Das, A. Das, M. Kundu, and M. Nasipuri, "A multi-scale deep quad tree based feature extraction method for the recognition of isolated handwritten characters of popular indic scripts", Pattern Recognition, Vol. 71, No. 11, pp. 78-93, 2017.

[6] S. Roy, N. Das, M. Kundu, and M. Nasipuri, "Handwritten isolated Bangla compound character recognition: A new benchmark using a novel deep learning approach", Pattern Recognition Letters, Vol. 90, No. 4, pp. 15-21, 2017.

[7] N. S. Panyam, T. R. V. Lakshmi, R. Krishnan, and N. V. K. Rao, "Modeling of palm leaf character recognition system using transform based techniques", Pattern Recognition Letters, Vol. 84, No.19, pp. 29-34, 2016.

[8] S. Jena, B. Majhi, and R. Mohapatra, "Twostage classification approach for the recognition of handwritten atomic Odia character", International Journal of Applied Pattern Recognition, Vol. 4, No. 1, p. 44, 2017.

[9] C. De Stefano, F. Fontanella, C. Marrocco, and A. Scotto di Freca, "A GA-based feature selection approach with an application to handwritten character recognition", Pattern Recognition Letters, Vol. 35, No. 1, pp. 130141, 2014.

[10] B. Singh, "Retina Recognition Using Support Vector Machine", International Journal of Engineering and Computer Science, Vol 5, No. 8, pp. 17605-17608, 2016.

[11] R. Mehta and K. Egiazarian, "Dominant Rotated Local Binary Patterns (DRLBP) for texture classification", Pattern Recognition Letters, Vol. 71, No. 3, pp. 16-22, 2016.

[12] K. Addakiri and M. Bahaj, "On-line Handwritten Arabic Character Recognition using Artificial Neural Network", International Journal of Computer Applications, Vol. 55, No. 13, pp. 42-46, 2012.

[13] V. Karbhari, D. Prapti, V. Sriniwas, and M. Majharoddin, "Zernike Moment Feature Extraction for Handwritten Devanagari (Marathi) Compound Character Recognition", International Journal of Advanced Research in
Artificial Intelligence, Vol. 3, No. 1, pp. 68-76, 2014.

[14] A. Abdalkafor, "Designing Offline Arabic Handwritten Isolated Character Recognition System using Artificial Neural Network Approach", International Journal of Technology, Vol. 8, No. 3, p. 528, 2017.

[15] P. Kamble and R. Hegadi, "Handwritten Marathi Character Recognition Using R-HOG Feature", Procedia Computer Science, Vol. 45, No. 1, pp. 266-274, 2015.

[16]P. Singh, R. Sarkar, N. Das, S. Basu, M. Kundu, and M. Nasipuri, "Benchmark databases of handwritten Bangla-Roman and DevanagariRoman mixed-script document images", Multimedia Tools and Applications, Vol. 76, No. 8, pp. 1-33, 2017.

[17] S. Naz, K. Hayat, M. Imran Razzak, M. Waqas Anwar, S. Madani, and S. Khan, "The optical character recognition of Urdu-like cursive scripts", Pattern Recognition, Vol. 47, No. 3, pp. 1229-1248, 2014.

[18] J. Olszewska, "Active contour based optical character recognition for automated scene understanding", Neurocomputing, Vol. 161, No. 20, pp. 65-71, 2015.

[19] O. Matei, P. Pop, and H. Vălean, "Optical character recognition in real environments using neural networks and k-nearest neighbor", Applied Intelligence, Vol. 39, No. 4, pp. 739748, 2013.

[20]M. Vaidya, Y. Joshi, and M. Bhalerao, "Marathi Numeral Identification System in Devanagari Script Using 1D Discrete Cosine Transform", International Journal of Intelligent Engineering and Systems, Vol. 10, No. 6, pp. 78-86, 2017.

[21] A.H. Gandomi, X.S. Yang, A.H. Alavi, "Mixed variable structural optimization using firefly algorithm", Computers \& Structures, Vol. 89, No. 23-24, pp. 2325-2336, 2011.

[22]R. Nakamura, L. Pereira, K. Costa, D. Rodrigues, J. Papa, and X. Yang, "BBA: A Binary Bat Algorithm for Feature Selection", In: Proc. of 25th SIBGRAPI Conference on Graphics, Patterns and Images, Ouro Preto, Brazil, pp. 291-297, 2012. 